\title{
Tendências Recentes de Mortalidade Cardiovascular nas Regiões de Saúde do Estado do Rio de Janeiro e Capital
}

\author{
Recent Trends in Cardiovascular Mortality in Rio de Janeiro State Health Regions and Capital
}

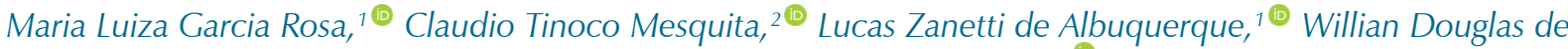 \\ Souza Silva, ${ }^{3}$ Vinicius de Padua Vieira Alves, ${ }^{1}$ Roger Freitas Ramirez Jordan, ${ }^{10}$ Ricardo Cardoso de Matos, ${ }^{1}$ Ana \\ Luisa Guedes de França e Silva, ${ }^{1}$ Erito Marques de Souza Filho ${ }^{3,4}{ }^{\circledR}$ \\ Universidade Federal Fluminense - Departamento de Epidemiologia e Bioestatística, ${ }^{1}$ Niterói, RJ - Brasil \\ Universidade Federal Fluminense Faculdade de Medicina - Departamento de Radiologia, ${ }^{2}$ Niterói, RJ - Brasil \\ Universidade Federal Fluminense - Departamento de Medicina Clínica, ${ }^{3}$ Niterói, RJ - Brasil \\ Universidade Federal Rural do Rio de Janeiro - Departamento de Tecnologias e Linguagens, ${ }^{4}$ Nova Iguaçu, RJ - Brasil
}

\section{Resumo}

Fundamento: A mortalidade por doenças cardiovasculares (DCV) vem mostrando tendência à estabilização em alguns países, incluindo o Brasil e o estado do Rio de Janeiro, após décadas de queda. Não encontramos análises detalhadas dessa tendência para o estado do Rio de Janeiro.

Objetivo: Analisar as tendências da mortalidade prematura e tardia por doenças do aparelho circulatório (DAC), doença isquêmica do coração (DIC) e doença cerebrovascular (DCBV) por sexo nas regiões de saúde do estado do Rio de Janeiro e capital (1996-2016).

Métodos: Dados de óbitos e população foram obtidos no DATASUS/MS. Taxas foram compensadas por códigos mal definidos, corrigidos pelos códigos cardiovasculares mal definidos e ajustadas por sexo e idade pelo método direto. $O$ Joinpoint Trend Analysis Software foi empregado para calcular a variação percentual anual (APC) e variação percentual anual média (AAPC). Foram consideradas para o estudo APC e AAPC significativamente diferentes de zero, calculadas por um teste de student com significância de $5 \%$.

Resultados: A mortalidade por DIC estabilizou ou até aumentou em pelo menos $50 \%$ das localidades analisadas (EAPC $\geq 0$ ). Nas regiões Norte e Noroeste, nenhuma mudança foi observada. Para DCBV, apenas uma região apresentou estabilidade na mortalidade (EAPC próximo a 0). Para as outras regiões, a taxa continuou a diminuir (APC <0) até 2016.

Conclusão: Esses resultados observados no Rio de Janeiro devem se repetir em várias regióes brasileiras e apontam para a necessidade de uma resposta na abordagem dos comportamentos no estilo de vida. Os médicos da atenção primária devem estar familiarizados com a tendência desfavorável da doença isquêmica do coração entre os adultos mais jovens e rastrear ativamente os fatores de risco para DCV, com atenção especial às mulheres. (Arq Bras Cardiol. $2021 ; 116(4): 763-771)$

Palavras-chave: Doenças Cardiovasculares/prevenção e controle; Doenças Cerebrovasculares/ prevenção e controle; Prevenção de Doenças; Redução; Fatores de Risco; Estilo de Vida; Epidemiologia.

\footnotetext{
Abstract

Background: Cardiovascular disease (CVD) mortality, after several decades of decrease, has shown a tendency towards the stabilization in some countries, including Brazil and Rio de Janeiro state. This new tendency was not further analyzed by gender, age group and region of the Rio de Janeiro state.

Objective: To analyze the trends of premature and late mortality from CVD, ischemic heart disease (IHD) and cerebrovascular disease (CBVD) by gender in the city of Rio de Janeiro (capital) and the health regions of Rio de Janeiro state (from 1996 to 2016.

Methods: Data on deaths and the population were obtained from DATASUS/MS. The rates were compensated by ill-defined codes, corrected by III-Defined Cardiovascular codes and gender and age-adjusted by the direct method (reference population - population of the state of Rio de Janeiro - 2000 census). The Joinpoint Trend Analysis Software was employed.
}

Correspondência: Roger Freitas Ramirez Jordan

Universidade Federal Fluminense - Epidemiologia e Bioestatistica - Rua Buarque de Macedo. CEP 24033-900, Rio de Janeiro, RJ - Brasil E-mail: rogerjordan6001@gmail.com

Artigo recebido em 25/10/2019, revisado em 14/02/2020, aceito em 16/03/2020

DOI: https://doi.org/10.36660/abc.20190742 
Results: IHD mortality stabilized or even increased for at least $50 \%$ of the analyzed areas (EAPC $\geq 0$ ). No change was observed. in the "North" and "Northwest" regions For CBVD, just one region showed stability regarding mortality (EAPC close to 0). For the other regions, the rate continued to decrease $(A P C<0)$ until 2016

Conclusion: These results observed in Rio de Janeiro are possibly appropriate to various Brazilian regions and demonstrate that a serious public health response is needed to address lifestyle behaviors. Primary care physicians should also be familiar with the unfavorable tendency in coronary heart disease among younger adults in recent years and actively screen for risk factors for cardiovascular disease, paying special attention to women. (Arq Bras Cardiol. 2021; 116(4):763-771)

Keywords: Cardiovascular Diseases/prevention and control; Cerebrovascular Diseases/prevention and control; Disease Prevention; Risk Factors; Life Style; Epidemiology.

Full texts in English - http://www.arquivosonline.com.br

\section{Introdução}

As doenças cardiovasculares (DCV) são uma das principais causas de morte prematura e incapacidade crônica no mundo e um grande obstáculo ao desenvolvimento humano sustentável, com um número estimado de 422,7 milhões de casos em todo o mundo em 2015, sendo 424.058 destes no Brasil. Em 2011, as Nações Unidas reconheceram formalmente doenças não transmissíveis, incluindo DCV, como uma grande preocupação para a saúde global.

As mudanças sociodemográficas nos últimos 25 anos foram associadas a declínios drásticos nas taxas de mortalidade padronizadas por idade por DCV em regiões com alto índice sociodemográfico, mas apenas uma diminuição gradual no resto do mundo, apesar dos avanços expressivos na capacidade técnica de prevenção e tratamento de DCV. ${ }^{1-4}$ Os dados do Global Burden of Disease (GBD) de 2015 mostraram redução da mortalidade por DCV padronizada por idade no Brasil e no estado Rio de Janeiro entre 1990 e 2015..$^{1-4}$

Na última década, estudos internacionais têm demonstrado uma tendência à estabilização dessas taxas..$^{5-7} \mathrm{~A}$ mesma tendência foi observada em alguns estados brasileiros, incluindo o Rio de Janeiro. ${ }^{4,8}$ No entanto, a estabilização dessa taxa de mortalidade não foi analisada por gênero, faixa etária ou regiões do estado, e essa desaceleração no declínio na mortalidade por DCV apresenta um novo desafio para as políticas de saúde em diferentes níveis de cobertura. O objetivo desse estudo é analisar as tendências da mortalidade prematura e tardia de doenças do aparelho circulatório (DAC), doenças isquêmicas do coração (DIC) e doenças cerebrovasculares (DCBV) por sexo na cidade e regiões de saúde do estado do Rio de Janeiro, entre 1996 e 2016, visando avaliar se houve mudanças recentes no padrão de declínio da mortalidade.

\section{Métodos}

Obtivemos dados de mortalidade e população para todos os indivíduos $\geq 20$ anos de idade das regiões de saúde e da capital do estado do Rio de Janeiro entre 1996 e 2016 no website do DATASUS/MS. ${ }^{8}$ De acordo com definição do Ministério da Saúde, uma região de saúde consiste em um espaço geográfico contínuo constituído por agrupamentos de municípios limítrofes, delimitado a partir de identidades culturais, econômicas e sociais e de redes de comunicação e infraestrutura de transportes compartilhados, com a finalidade de integrar a organização, o planejamento e a execução de ações de serviços de saúde. ${ }^{9}$
A mortalidade foi categorizada em prematura (30 a 69 anos) e tardia (70 anos ou mais) e foi analisada por sexo e causa para a capital e regiões de saúde do estado do Rio de Janeiro.10 Consideramos os códigos listados no capítulo IX da CID-10 para DAC e, especificamente, os códigos I20-I25 para DIC e I60-I69 para DCBV da CID-10. As taxas de mortalidade bruta e ajustada por sexo e idade (pelo método direto) foram calculadas por 100.000 habitantes, para cada localidade. Atribuímos as mortes por causas mal definidas - códigos do CID-10 RR00-RR99 - às frações observadas nas mortes definidas por DAC, DIC e DCBV (mortes compensadas). Os códigos cardiovasculares mal definidos - 146, 147, 148, 149, 150, 151 e 170 - foram atribuídos à causa DIC, por sexo e categoria de idade.11 Após essas correções, foram estimadas as taxas de mortalidade prematura e tardia ajustadas ao sexo e idade, sempre para cada causa, sexo e localidade. A população de referência foi a população do estado do Rio de Janeiro (censo de 2000), estratificada em sete faixas etárias (20 a 29 anos; 30 a 39 anos; 40 a 49 anos, 50 a 59 anos; 60 a 69 anos; 7079 anos e 80 anos ou mais) para cada sexo. Essas taxas foram denominadas compensadas e ajustadas.

\section{Análise Estatística}

Para avaliar as tendências, foi empregado o Joinpoint Trend Analysis Software, versão 4.7.0.0. ${ }^{12} \mathrm{O}$ modelo de regressão do Joinpoint Poisson calcula a variação percentual anual (APC) a partir da taxa de mortalidade bruta e ajustada. Então, a variação percentual anual média (AAPC) é estimada como o peso médio das APC, em que os períodos são utilizados como peso. Posteriormente, é testado se as APC ou a AAPC são significativamente diferentes de zero por meio de um teste $t$ de Student não pareado com significância de $5 \%$. O número de joinpoints foi calculado pelo teste de permutação, utilizando as correções de Bonferroni, em que o nível alfa ajustado foi de $5 \%$. O software supõe que o valor padrão do número mínimo de ponto de junção seja 0 . Tal método é preferido para produzir resultados mais parcimoniosos.

\section{Resultados}

Em todo o período de 1996 a 2016, a taxa de mortalidade prematura e tardia ajustada à idade para adultos com idade $\geq 20$ anos declinou aproximadamente à metade em todas as regiões do estado do Rio de Janeiro e capital, nos três grupos estudados (DCV, DIC e DCBV), para ambos os sexos quando a AAPC variou de $0,6 \%$ a $6,5 \%$. Em geral, a mortalidade 
prematura para homens foi maior que para as mulheres para as três causas, o que não se repetiu para mortalidade tardia. Os coeficientes para mortalidade tardia foram pelo menos 10 vezes maiores que os da mortalidade prematura, tanto para homens e mulheres, quanto para as três causas (Tabela 1).

Analisamos as regiões do estado do Rio de Janeiro e a capital no período de 1996 a 2016. Para ambos os sexos, a mortalidade prematura por DIC estabilizou ou até reverteu a tendência em pelo menos $50 \%$ das localidades analisadas (APC $>0$ ou $A P C \cong 0$ ). O mesmo aconteceu com a mortalidade tardia por DIC para as mulheres. Essa mudança ocorreu em diferentes momentos entre 2003 e 2014. Na região metropolitana I, a DIC prematura e tardia para homens e mulheres aumentou a partir 2008 ou 2013. Nas regiões Norte e Noroeste, nenhuma mudança foi observada. Em outras regiões e na capital do Rio de Janeiro, houve mudança na tendência (APC $\geq 0$ ) para alguns grupos (Tabelas 2 e 3 ).

Para DCBV, apenas uma região (Serrana) apresentou estabilidade na mortalidade prematura (APC $\cong 0 \%$ ). Para as demais regiões, tanto para mortalidade prematura quanto tardia, para homens e mulheres, a taxa continuou a diminuir (APC <0) até 2016 (ver Tabelas 2 e 3).

Em todo o Capítulo IX (CID-10), DAC, observamos uma mudança na tendência da mortalidade prematura em quatro das dez regiões analisadas para homens (Rio de Janeiro capital, Centro-Sul, Médio Paraíba e Metropolitana 1) e em três regiões para mulheres (Médio Paraíba, Metropolitana 1 e Metropolitana 2). Quanto à mortalidade tardia, ocorreram mudanças na tendência da mortalidade em três regiões para mulheres (Rio de Janeiro capital, Metropolitana 1 e Metropolitana 2) e uma para homens (Metropolitana 1). Os anos em que essa mudança ocorreu variaram de 2001 a 2012. A região Metropolitana I apresentou o maior número de mudanças na tendência de mortalidade (ver Tabelas 2 e 3).

É importante ressaltar que, em nenhum dos casos em que houve aumento na mortalidade, essa mudança foi estatisticamente significativa.

\section{Discussão}

A presente análise teve como objetivo identificar mudanças na tendência de diminuição da mortalidade cardiovascular precoce e tardia nas regiões de saúde do estado do Rio de Janeiro e capital, entre 1996 e 2016, de acordo com o sexo.

Pelo menos 50\% das localidades mostraram mudanças na mortalidade por DIC, estabilizando ou aumentando a taxa, principalmente para mortalidade prematura em homens e para ambas as mortalidades para mulheres. Para DAC, os resultados foram semelhantes. Esses resultados são particularmente desafiadores, pois a mortalidade prematura impõe um alto ônus social e confirma a evidência de que, no tocante às doenças isquêmicas do coração, as mulheres são subdiagnosticadas e subtratadas, levando ao aumento das complicações e da taxa de mortalidade. ${ }^{13}$

Em uma escala global, a taxa de mortalidade média por DAC ajustada para idade continuamente seguiu um padrão de redução nos anos 1990 e 2000, com o maior declínio entre 2000 e 2005. Isso contou principalmente na redução das taxas de mortalidade por DIC e DCBV, essa última também apresentando a maior queda percentual nos coeficientes de mortalidade prematuro e tardio em todas as regiões de saúde do Rio de Janeiro e capital. ${ }^{14}$ Além disso, regiões de baixa e média renda ao redor do mundo apresentaram uma queda de $13 \%$ na taxa de mortalidade ajustada para idade atribuída à DAC, mas com um expressivo aumento de $66 \%$ no número total de mortes por essa causa entre 1990 e $2013 .{ }^{14}$ Apesar disso, esse aumento de mortes por DAC atribuídas ao crescimento e envelhecimento populacional foi compensado pela redução em taxas de mortalidade idade-específicas no Brasil, o que aponta para uma possível melhora na qualidade de saúde da população. ${ }^{15}$ No entanto, a distribuição global das DAC é complexa, influenciada por características nacionais e regionais, que resultam em importantes diferenças entre e dentro de regiões, tornando difícil realizar comparações.

Nos EUA, a taxa de declínio para todas as DCV desacelerou substancialmente após 2011. ${ }^{5}$ Argumenta-se que, em parte, o declínio se deve à crescente prevalência de obesidade e diabetes em proporções epidêmicas nos últimos anos, superando os benefícios das políticas de prevenção, os efeitos da prevenção primária e os avanços no tratamento de hipertensão, diabetes e dislipidemia. ${ }^{1,3,16}$ No Brasil, embora a cobertura do sistema nacional de vigilância de mortalidade tenha expandido e a qualidade dos certificados de óbito aumentado, com maior proporção de diagnósticos corretos, ${ }^{17}$ não se observa o mesmo para a avaliação da prevalência de fatores de risco. A única fonte de dados seriados sobre fatores de risco cardiovasculares no Brasil é o Vigitel, que cobre apenas capitais. Trata-se de uma pesquisa telefônica anual "nacional", criticada por alguns por serem autorrelatados. A partir de dados coletados em 2006 a 2016, observamos, para a capital do Rio de Janeiro, aumento na hipertensão autorreferida e obesidade, mas diminuição no tabagismo. ${ }^{18}$

Outros estudos de revisão, como o realizado por Picon et al, estimam taxas de prevalência para hipertensão arterial no Brasil de 28,7\% (95\% intervalo de confiança, 26,2-31,4) para ambos os sexos em todas as regiões federativas. ${ }^{19} \mathrm{Um}$ número considerável de outros estudos sobre a prevalência de fatores de risco cardiovasculares no Brasil pode ser encontrado na literatura, mas, em sua maioria, observam-se limitações metodológicas referentes à cobertura espacial e ausência de exames laboratoriais confirmatórios. ${ }^{20}$

Nas regiões do Rio de Janeiro, foram observadas mudanças nas tendências de mortalidade por DIC e DAC, com estabilização ou até mesmo aumento das taxas entre 2004 e 2014. Descreve-se que as primeiras evidências de uma estabilização e sinais precoces de aumento entre jovens adultos nos EUA, embora sem significância estatística, foram publicadas pela primeira vez em 2007. ${ }^{21}$ Muitos fatores podem se relacionar com essa tendência regional observada no Rio de Janeiro, e dados que mostram melhora na qualidade de saúde e de vida tornam difícil compreender a razão para essa tendência de achatamento observada. ${ }^{22}$ A cobertura do sistema de saúde suplementar no estado do Rio de Janeiro em 2013 era de 33,5\%, com aumento anual de 0,78\% entre 2004 e 2013. Correlações lineares inversas com taxas de mortalidade por DAC e DCBV foram observadas, o que deve ser questionado pela possibilidade de outros fatores 
参

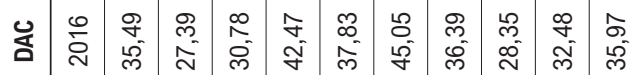

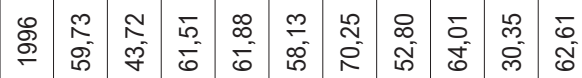

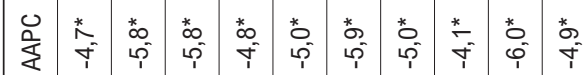

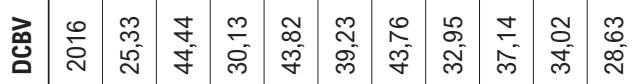

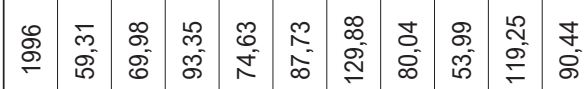

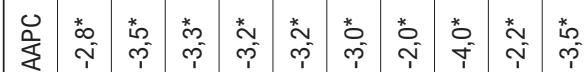

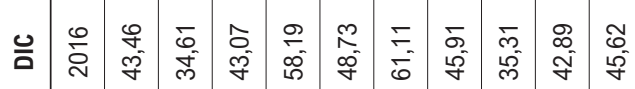

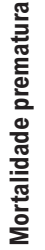

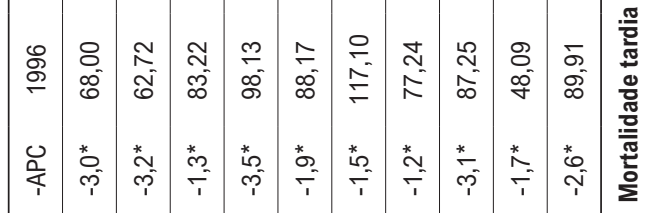

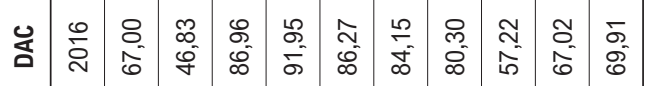

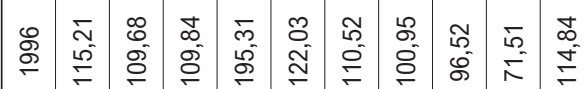

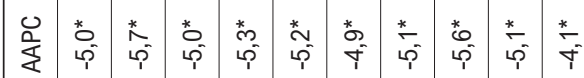

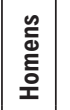

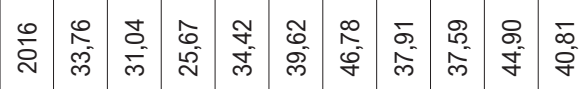

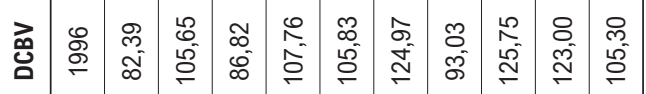

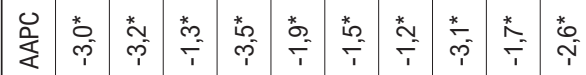

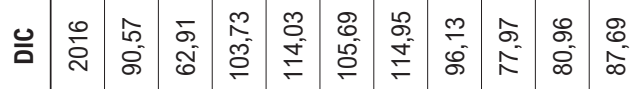

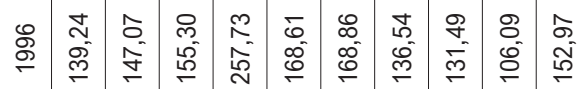

产产

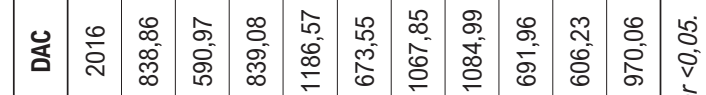

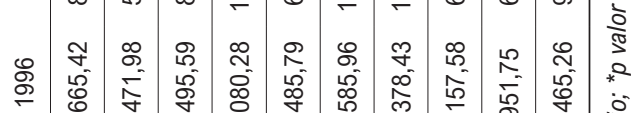

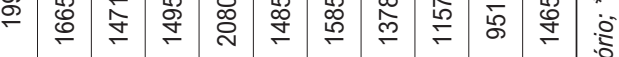

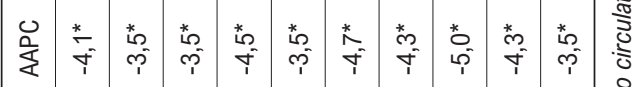

>

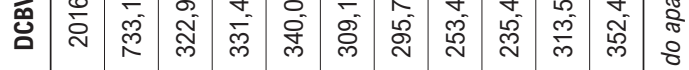

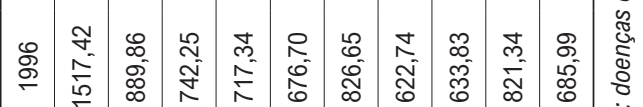

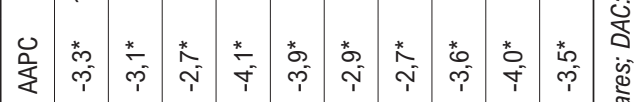

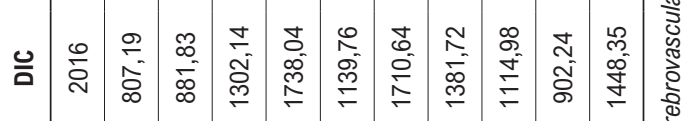

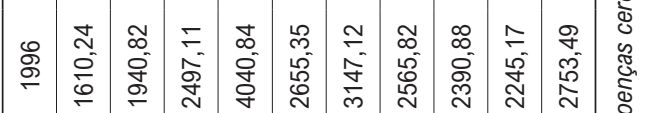

茓

L)

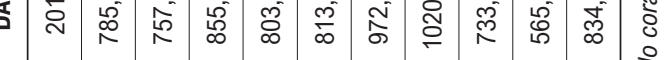

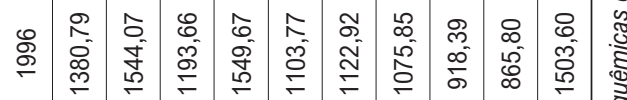

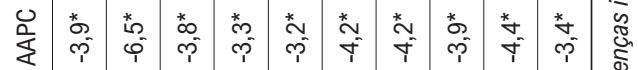

俤

产

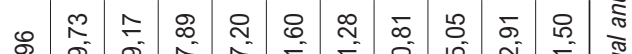

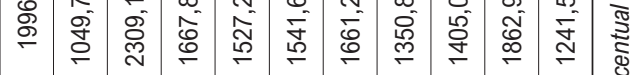

旁等

- 0 ก

U

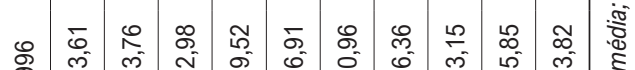

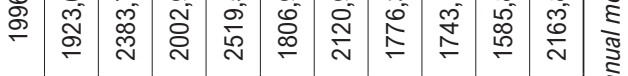
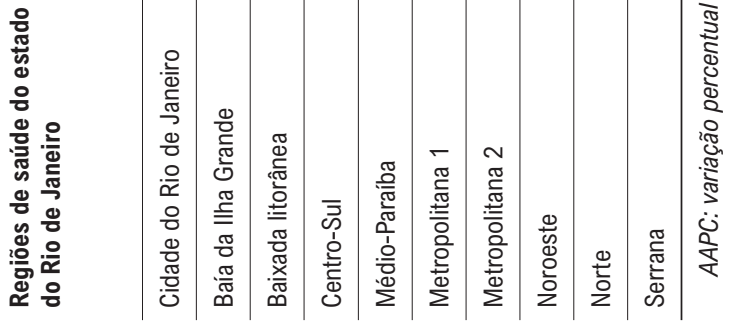
Tabela 2 - Mudança percentual anual (APC) na mortalidade cardiovascular prematura na cidade do Rio de Janeiro (capital) e regiões de saúde do estado do Rio de Janeiro (1996-2016)

\begin{tabular}{|c|c|c|c|c|c|}
\hline \multicolumn{6}{|c|}{ Mortalidade prematura } \\
\hline \multirow{2}{*}{$\begin{array}{l}\text { Grupo de } \\
\text { doenças }\end{array}$} & \multirow{2}{*}{ Região de saúde e capital } & \multicolumn{2}{|c|}{ Homens } & \multicolumn{2}{|c|}{ Mulheres } \\
\hline & & Período & APC & Período & APC \\
\hline & & $1996-2003$ & $-4,2^{*}$ & $1996-2000$ & $-9,5^{*}$ \\
\hline & & 2003-2008 & $-0,8$ & $2000-2016$ & $-1,1^{*}$ \\
\hline & 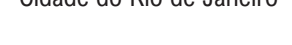 & $2008-2014$ & $-3,4^{*}$ & & \\
\hline & & $2014-2016$ & 4,2 & & \\
\hline & Baía da Ilha Grande & & & $1996-2010$ & $-7,7^{*}$ \\
\hline & & & & $2010-2016$ & 7,2 \\
\hline & Baixada litorânea & & & 1996-1998 & $-24,8^{*}$ \\
\hline & & & & 1998-2004 & 3,4 \\
\hline & & & & 2004-2008 & $-6,9$ \\
\hline & & & & $2008-2016$ & $-0,3$ \\
\hline \multirow[t]{17}{*}{ DIC } & Centro-Sul & 1996-2007 & $-6,8^{*}$ & & \\
\hline & & 2007-2016 & 0,2 & & \\
\hline & Médio-Paraíba & $1996-2010$ & $-4,2^{*}$ & $1996-2010$ & $-5,3^{*}$ \\
\hline & & $2010-2016$ & 1,7 & $2010-2016$ & 1,8 \\
\hline & Metropolitana 1 & 1996-2009 & $-3,7^{*}$ & 1996-2008 & $-5,5^{*}$ \\
\hline & & $2009-2016$ & 2,0 & $2008-2016$ & 0,8 \\
\hline & Metropolitana 2 & 1996-2009 & $-2,7^{*}$ & 1996-2009 & $-4,1^{*}$ \\
\hline & & $2009-2016$ & 0,4 & $2009-2016$ & 2,0 \\
\hline & Noroeste & & & & \\
\hline & Norte & & & & \\
\hline & Serrana & & & & \\
\hline & Cidade do Rio de Janeiro & & & & \\
\hline & Baía da Ilha Grande & & & & \\
\hline & Baixada litorânea & 1996-2005 & $-2,3$ & & \\
\hline & & $2005-2016$ & $-7,2^{*}$ & & \\
\hline & Centro-Sul & & & & \\
\hline & Médio-Paraíba & & & & \\
\hline \multirow[t]{7}{*}{ DCBV } & Metropolitana 1 & $1996-2013$ & $-5,5^{*}$ & & \\
\hline & & 2013-2016 & $-1,8$ & & \\
\hline & Metropolitana 2 & & & & \\
\hline & Noroeste & & & & \\
\hline & Norte & & & & \\
\hline & Serrana & 1996-2009 & $-6,3^{*}$ & & \\
\hline & & $2009-2016$ & -0 & & \\
\hline
\end{tabular}




\begin{tabular}{|c|c|c|c|c|c|}
\hline \multicolumn{6}{|c|}{ Continuação } \\
\hline \multirow{20}{*}{ DAC } & Cidade do Rio de Janeiro & $1996-2003$ & $-4,7^{*}$ & $1996-2000$ & $-8,9^{*}$ \\
\hline & & $2003-2008$ & $-1,2$ & $2000-2016$ & $-1,6^{*}$ \\
\hline & & $2008-2014$ & $-5,1^{*}$ & & \\
\hline & & $2014-2016$ & 5,8 & & \\
\hline & Baía da llha Grande & & & & \\
\hline & Baixada litorânea & & & 1996-1998 & $-27,3^{*}$ \\
\hline & & & & $1998-2003$ & 4,6 \\
\hline & & & & 2003-2016 & $-2,7^{*}$ \\
\hline & Centro-Sul & 1996-2005 & $-7,5^{*}$ & & \\
\hline & & $2005-2016$ & -0 & & \\
\hline & Médio-Paraíba & 1996-2012 & $-3,6^{*}$ & $1996-2010$ & $-5,0^{*}$ \\
\hline & & $2012-2016$ & 5,2 & $2010-2016$ & 1,7 \\
\hline & Metropolitana 1 & $1996-2006$ & $-3,0^{*}$ & $1996-2008$ & $-3,7^{*}$ \\
\hline & & $2006-2016$ & 0 & $2008-2016$ & 0,5 \\
\hline & Metropolitana 2 & & & $1996-2007$ & $-3,6^{*}$ \\
\hline & & & & $2007-2016$ & 1,2 \\
\hline & Noroeste & & & & \\
\hline & Norte" & & & & \\
\hline & Serrana & & & & \\
\hline & Serrana & & & & \\
\hline
\end{tabular}

APC: mudança percentual anual; DIC: doenças isquêmicas do coração; DCBV: doenças cerebrovasculares; DAC: doenças do aparelho circulatório; ${ }^{*} p$ valor< 0,05. Linhas vazias: não foi observada mudança de tendência.

simultâneos - como o aumento no índice de desenvolvimento humano municipal em todas as unidades federativas entre 2000 e 2010 - também terem afetado essas taxas. ${ }^{21}$

Conforme discutido por Soares et al. em dois trabalhos diferentes, melhoras socioeconômicas precederam o declínio na mortalidade por DCV. ${ }^{23,24} \mathrm{~A}$ redução nas taxas de mortalidade, principalmente por DIC no estado do Rio de Janeiro nas últimas décadas, foi precedida por um aumento no índice de desenvolvimento humano (IDH). ${ }^{23}$ Apenas poucos municípios do estado do Rio de Janeiro tiveram queda no IDH entre 1970 e 1991. Alguns deles estão localizados nas regiões norte e nordeste, onde a mortalidade por DIC está caindo continuamente. Essas observações permitem levantar a hipótese de que esses municípios estão se beneficiando das melhoras socioeconômicas que ocorreram anteriormente em regiões onde há uma inversão na tendência da mortalidade, chamando a atenção para a Metropolitana I.

Nos EUA, o declínio em toda a mortalidade por DCV desacelerou substancialmente, incluindo a DCBV, o que não ocorreu nas regiões de saúde e capital do Rio de Janeiro. ${ }^{2}$ Isso pode ser explicado pelo atraso na implementação de medidas primárias de prevenção de acidente vascular encefálico (AVE) isquêmico (estatinas, ácido acetilsalicílico, terapia antitrombótica) em comparação com países desenvolvidos. Em escala nacional, o achatamento do padrão das taxas de mortalidade por DAC observados nos anos recentes serve como sinal de alerta para a necessidade de monitoramento contínuo e a possibilidade da emergência de contratendências significativas $\mathrm{G} .{ }^{2} \mathrm{O}$ sistema de saúde público, com programas como a estratégia de saúde da família e programa da farmácia popular, tem importância indubitável no controle da hipertensão e outros fatores de risco cardiovascular. ${ }^{25,26}$ Campanhas nacionais efetivas antitabaco, planos nacionais de prevenção e controle da obesidade e outras políticas com objetivo de monitorar e reduzir fatores de risco fizeram parte da história recente do desenvolvimento do sistema de saúde público brasileiro. ${ }^{27-29}$ É crucial entender os mecanismos que podem estar prejudicando sua eficiência.

\section{Limitações}

O presente estudo tem algumas limitações. Uma delas é a qualidade das declarações de óbito, que não é uniforme entre os municípios e tem variado ao longo do tempo, o que pode ter afetado as tendências observadas. A segunda limitação é a existência de municípios que compõem as regiões de saúde com pequenas populações, o que leva a grandes 
Tabela 3 - Mudança percentual anual (APC) na mortalidade cardiovascular tardia na cidade do Rio de Janeiro (capital) e regiões de saúde do estado do Rio de Janeiro (1996-2016)

\section{Mortalidade Tardia}

\begin{tabular}{|c|c|c|c|c|c|}
\hline \multirow{2}{*}{ Grupo de doenças } & \multirow{2}{*}{ Regiões de saúde e capital } & \multicolumn{2}{|c|}{ Homens } & \multicolumn{2}{|c|}{ Mulheres } \\
\hline & & Período & APC & Período & APC \\
\hline \multirow{17}{*}{ DIC } & Cidade do Rio de Janeiro & & & $1996-2011$ & $-5,4^{*}$ \\
\hline & & & & 2011-2016 & 3,3 \\
\hline & Baía da Ilha Grande & & & & \\
\hline & Baixada litorânea & & & & \\
\hline & Centro-Sul & & & $1996-2010$ & $-6,7^{*}$ \\
\hline & & & & 2010-2016 & 2,2 \\
\hline & Médio-Paraíba & & & & \\
\hline & Metropolitana 1 & 1996-2001 & $-7,8^{*}$ & $1996-2008$ & $-6,0^{*}$ \\
\hline & & $2001-2013$ & $-1,9^{*}$ & 2008-2016 & 1,9 \\
\hline & & 2013-2016 & 7,6 & & \\
\hline & Metropolitana 2 & & & $\begin{array}{l}1996- \\
2010\end{array}$ & $-5,4^{*}$ \\
\hline & & & & 2010-2016 & 3,7 \\
\hline & Noroeste & & & & \\
\hline & Norte & & & & \\
\hline & Serrana & & & $1996-2005$ & $-3,1^{*}$ \\
\hline & & & & 2005-2009 & $-9,3$ \\
\hline & & & & 2009-2016 & $-0,7$ \\
\hline \multirow{12}{*}{ DCBV } & Cidade do Rio de Janeiro & 1996-2004 & $-1,8^{*}$ & & \\
\hline & & 2004-2016 & $-5,2^{*}$ & & \\
\hline & Baía da Ilha Grande & 1996-1998 & $-43,4^{*}$ & & \\
\hline & & 1998-2016 & $-1,1$ & & \\
\hline & Baixada litorânea & & & & \\
\hline & Centro-Sul & & & & \\
\hline & Médio-Paraíba & & & & \\
\hline & Metropolitana 1 & & & & \\
\hline & Metropolitana 2 & & & & \\
\hline & Noroeste & & & & \\
\hline & Norte & & & & \\
\hline & Serrana & & & & \\
\hline \multirow{15}{*}{ DAC } & Cidade do Rio de Janeiro & & & $1996-2012$ & $-5,1^{*}$ \\
\hline & & & & $2012-2016$ & 2,9 \\
\hline & Baía da Ilha Grande & & & & \\
\hline & Baixada litorânea & & & & \\
\hline & Centro-Sul & & & & \\
\hline & Médio-Paraíba & & & & \\
\hline & Metropolitana 1 & 1996-2001 & $-6,2^{*}$ & $1996-2001$ & $-7,8^{*}$ \\
\hline & & $2001-2016$ & 0,1 & $2001-2016$ & $-0,5$ \\
\hline & Metropolitana 2 & & & 1996-2006 & $-2,2^{*}$ \\
\hline & & & & 2006-2009 & $-10,4$ \\
\hline & & & & 2009-2016 & $6,3^{*}$ \\
\hline & Noroeste & & & 1996-2012 & $-1,2$ \\
\hline & & & & 2012-2016 & $-11,2^{*}$ \\
\hline & Norte & & & & \\
\hline & Serrana & & & & \\
\hline
\end{tabular}

APC: mudança percentual anual; DIC: doenças isquêmicas do coração; DCBV: doenças cerebrovasculares; DAC: doenças do aparelho circulatório; *p valor< 0,05. Linhas vazias: não foi observada mudança de tendência. 
oscilações na ocorrência de eventos pouco frequentes, como a morte. A terceira limitação é a ausência de informações sobre a prevalência dos fatores de risco cardiovascular para as localidades analisadas.

\section{Conclusão}

As tendências adversas de mortalidade por DIC e DAC em homens adultos jovens e mulheres nas duas faixas etárias foram observadas em $50 \%$ das regiões de saúde no estado do Rio de Janeiro. Esses resultados observados no Rio de Janeiro devem se repetir em várias regiões brasileiras, e apontam para a necessidade de uma resposta na abordagem dos comportamentos no estilo de vida. Os médicos da atenção primária devem estar familiarizados com a tendência desfavorável da doença isquêmica do coração entre os adultos mais jovens e rastrear ativamente os fatores de risco para DCV, com atenção especial às mulheres.

\section{Contribuição dos Autores}

Concepção e desenho da pesquisa: Rosa MLG, Mesquita CT, Albuquerque LZ, Silva WDS, Alves VPV, Matos RC, Souza Filho EM; Obtenção de dados, Análise estatística, Redação do manuscrito: Rosa MLG, Mesquita CT, Albuquerque LZ, Silva WDS, Alves VPV, Jordan RFR, Matos

\section{Referências}

1. Sidney S, Quesenberry CP Jr, Jaffe MG,Sorel M, Huynh M, Kusbi L, et al. Recent trends in cardiovascular mortality in the United States and public health goals. JAMA Cardiol. 2016;1(5):594-9.

2. Nowbar AN, Gitto M, Howard JP,Francis DP, Lamee RA. Mortality from ischemic heart disease. Circ Cardiovasc Qual Outcomes. 2019;12(6):e005375.

3. O'Flaherty M, Allender S, Taylor R,Stevenson C, Peeters A, Capewell S. The decline in coronary heart disease mortality is slowing in young adults (Australia 1976-2006): a time trend analysis. Int J Cardiol. 2012;158(2):193-8.

4. Brant LCC, Nascimento BR, Passos VMA, Duncan BB, Bensenor IJM, Malta DC, et al. Variations and particularities in cardiovascular disease mortality in Brazil and Brazilian states in 1990 and 2015: estimates from the Global Burden of Disease. Rev Bras Epidemiol. 2017;20(Suppl 1):116-28.

5. Roth GA, Johnson C, Abajobir A, Abd-Allah F, Abera SF, Abyu G, et al. Global, regional, and national burden of cardiovascular diseases for 10 causes, 1990 to 2015. J Am Coll Cardiol. 2017;70(11):1-25.

6. Lotufo PA. The pace of reduction of cardiovascular mortality in Brazil (1990 to 2017) is slowing down. Sao Paulo Med J. 2019;137(1):3-5.

7. Brant LCC, Nascimento BR, Passos VMA,. Duncan BB, Bensenor IJM, Malta $D$, et al. Variations and particularities in cardiovascular disease mortality in Brazil and Brazilian states in 1990 and 2015: estimates from the Global Burden of Disease. Rev Bras Epidemiol. 2017;20(Suppl 1):116-28.

8. Brasil. Ministério da Saúde. DATASUS. Informações de Saúde. Estatísticas Vitais. [Acesso em agosto de 2019]. Disponível em:http://datasus.saude. gov.br/

9. Brasil. Ministério da Saúde Comissão Intergestores Tripartite. Resolução no 1, de 29 de setembro de 2011. [Acesso em 14/02/2020]. Na rede mundial de computadores: Disponível em:http://bvsms.saude.gov.br/bvs/saudelegis/ cit/2011/res0001_29_09_2011.html.
RC, Silva ALGF, Souza Filho EM; Análise e interpretação dos dados: Rosa MLG, Mesquita CT, Albuquerque LZ, Matos RC, Souza Filho EM; Obtenção de financiamento: Albuquerque LZ; Revisão crítica do manuscrito quanto ao conteúdo intelectual importante: Rosa MLG, Mesquita CT, Albuquerque LZ, Silva WDS, Alves VPV, Jordan RFR, Souza Filho EM.

\section{Potencial Conflito de Interesses}

Os autores declaram não haver conflito de interesses pertinentes.

\section{Fontes de Financiamento}

O presente estudo não contou com fontes de financiamento externas.

\section{Vinculação Acadêmica}

Não há vinculação deste estudo a programas de pósgraduação.

\section{Aprovação Ética e Consentimento Informado}

Este artigo não contém estudos com humanos ou animais realizados por nenhum dos autores.

10. World Health Organization. (WHO). WHO Global NCD Action Plan $2013-$ 2020 [Internet]. Genebra: World Health Organization, 2013 [acessado em 12 out. 2019]. Disponível em: https://www.who.int/nmh/events/ ncd_action_plan/en/.

11. Soares DA, Gonçalves MJ. Mortalidade cardiovascular e impacto de técnicas corretivas de subnotificações e óbitos mal definidos. Rev Panam Salud Publica. 2012;32(3):199-206.

12. National Institute of Health. National Cancer Institute. Joinpoint Trend Analysis Software.[Citado em 12 out 2019]. Disponível em: https:// surveillance.cancer.gov/joinpoint/

13. Rosen SE, Henry S, Bond R,Pearte T, Mieres JH. Sex-specific disparities in risk factors for coronary heart disease. Curr Atheroscler Rep. 2015; 17(8):49.

14. Roth GA, Huffman MD, Moran AE,Feigin V, Mensah GA, Naghavi M, et al. Global and regional patterns in cardiovascular mortality from 1990 to 2013. Circulation. 2015;132(17):1667-78

15. Roth GA, Forouzanfar MH, Moran AE,Barber R, Nguyen G, Feigin VL, et al Demographic and epidemiologic drivers of global cardiovascular mortality. N Engl J Med. 2015; 372(14):1333-41.

16. Wilmot KA, O'Flaherty M, Capewell S, Ford ES, Vaccarino V. Coronary heart disease mortality declines in the United States from 1979 through 2011: evidence for stagnation in young adults, especially women. Circulation. 2015; 132:997-1002.

17. França EB, Passos VMA, Malta DC,Abreu D, Vasconcelos AM, Carneiro M, et al. Cause-specific mortality for 249 causes in Brazil and states during 19902015: a systematic analysis for the global burden of disease study 2015. Popul Health Metr. 2017;15(1):39.

18. Vigitel Brasil 2016: vigilância de fatores de risco e proteção para doença crônicas por inquérito telefônico: estimativas sobre frequência e distribuição sociodemográfica de fatores de risco e proteção para doenças crônicas nas capitais dos 26 estados brasileiros e no Distrito Federal em 2016. Brasília, 2017. 


\section{Artigo Original}

19. Picon RV, Fuchs FD, Moreira LB, Riegel G, Fuchs SC. Trends in prevalence of hypertension in Brazil: a systematic review with meta-analysis. PLoS One. 2012; 7(10):e48255.

20. Ribeiro AL, Duncan BB, Brant LC,Lotufo P, Barreto SM. Cardiovascular health in Brazil: trends and perspectives. Circulation. 2016; 133(4):422-33.

21. Ford ES, Capewell S. Coronary heart disease mortality among young adults in the U.S. from 1980 through 2002: concealed leveling of mortality rates. J Am Coll Cardiol. 2007;50(22):2128-32.

22. Lotufo PA. The pace of reduction of cardiovascular mortality in Brazil (1990 to 2017) is slowing down. São Paulo Med J. 2019;137(1):3-5.

22. Villela PB, Klein $\mathrm{CH}$, de Oliveira GMM. Socioeconomic factors and mortality due to cerebrovascular and hypertensive disease in Brazil. Rev Port Cardiol. 2019; 38(3):205-12.

23. Soares GP, Klein CH, Silva NA Progression of mortality due to diseases of the circulatory system and human development index in Rio de Janeiro Municipalities. Arq Bras Cardiol. 2016 Oct; 107(4):314-22.
24. Soares GP, Brum JD, Oliveira GM, Oliveira GMM, Klein CH, Silva NAS. Allcause and cardiovascular diseases mortality in three Brazilian states, 1980 to 2006. Rev Panam Salud Publica. 2010; 28(4):258-66.

25. Ceccon RF, Borges DO, Paes LG,Klafke JZ, Viecili PRN. Mortality due to circulatory disorders and the evolution of family health in Brazil: an ecological study. Cien Saude Colet. 2013;18(5):1411-6.

26. Santos-Pinto Cdu B, Costa Ndo R, Osorio-de-Castro CG. The "Farmácia Popular do Brasil" Program and aspects of public provision of medicines in Brazil. Cien Saude Colet. 2011;16(6):2963-73.

27. Levy D, de Almeida LM, Szklo A. The Brazil SimSmoke policy simulation model: the effect of strong tobacco control policies on smoking prevalence and smoking-attributable deaths in a middle income nation. PLoS Med. 2012; 9(11):e1001336.

28. Jaime PC, da Silva AC, Gentil PC, Claro RM, Monteiro CA. Brazilian obesity prevention and control initiatives. Obes Rev. 2013;14(Suppl 2):88-95.

29. Paim J, Travassos C, Almeida C,Macinko BL. The Brazilian health system: history, advances, and challenges. Lancet. 2011;377(9779):1778-97. 